\title{
Effect of a Low-conductivity Zone on Field-amplified Sample Stacking in Microchip Micellar Electrokinetic Chromatography
}

\author{
Kenji Sueyoshi, ${ }^{\dagger}$ Fumihiko Kitagawa, and Koji Otsuka \\ Department of Material Chemistry, Graduate School of Engineering, Kyoto University, Katsura, Nishikyo, \\ Kyoto 615-8510, Japan
}

\begin{abstract}
The effects of a low-conductivity (low-C) zone of an on-line sample preconcentration based on field-amplified sample stacking (FASS) under an acidic condition was investigated in microchip micellar electrokinetic chromatography (MCMEKC). By employing originally fabricated microchips with $\mathrm{T}$ - and cross-channel injectors on a single device, partial injection of the low-C zone and large-volume injection of a sample solution were demonstrated. As a typical result, the observed peak intensities of the fluorescent dyes were 1.5-2.0-fold enhanced by partial injection of the low-conductivity zone, as compared to those in FASS-MCMEKC without injecting the low-C zone; also, 30 - 40-fold enhancements of the detectability were achieved as compared to the conventional MCMEKC analyses. The resolutions of the hydrophobic analytes were also improved by introducing the low-C zone. The difference in the retention factors of model analytes in the high-C background and low-C zones was also estimated in order to clarify the effect of the low-C zone on the resolution and detectability in FASS-MCMEKC.
\end{abstract}

(Received October 8, 2012; Accepted November 14, 2012; Published January 10, 2013)

\section{Introduction}

Recently, microchip electrophoresis (MCE) has attracted the attention of scientists, since MCE can resolve various compounds with a short analysis time, high resolution, and small consumptions of samples. ${ }^{1-5}$ However, the sensitivity in MCE equipped with an optical detection scheme is sometimes not sufficient for analyses of diluted samples, because of the short optical pathlength of the microchannels. Therefore, various online sample preconcentration techniques have been developed to improve the sensitivity of MCE. ${ }^{6-10}$ One of the successful strategies is to combine other preconcentration devices with MCE on a single microchip, e.g., solid-phase extraction, ${ }^{11}$ filtering using a nanopore or nanochannel, ${ }^{12,13}$ and so on. ${ }^{14,15}$ As a typical example, Kim et al. reported that the preconcentration with a nanochannel could attain a million-fold improvement of the detectability, but it required a long analysis time of over 30 min. ${ }^{13}$ Furthermore, the nano-fabrication schemes need an expensive apparatus and complicated procedures. Thus, it is appropriate for MCE to apply a rapid and simple preconcentration technique including field-amplified stacking (FASS), ${ }^{16-18}$ sweeping, ${ }^{19-21}$ transient isotachophoresis, ${ }^{22,23}$ isoelectric focusing, ${ }^{24,25}$ transient trapping, ${ }^{26}$ and so on. Especially, stacking techniques have often been applied to capillary electrophoresis and MCE, since it is easy to use. ${ }^{16-18}$ These stacking techniques are based on a difference in the electric fields between a sample solution (S) and background solution (BGS) zones filled in a microchannel. Briefly, the S and BGS solutions are prepared with low- and high-conductivity solutions, respectively. When the voltage is applied to a microchannel filled with a

† To whom correspondence should be addressed.

E-mail: sueyoshi@anchem.mc.kyoto-u.ac.jp high-conductive BGS and low-conductive $S$, a high electric field is generated in the $\mathrm{S}$ zone, as compared to that in the BGS zone. Thus, the difference in the migrating velocity of ionic analytes between the $\mathrm{S}$ and BGS zones occurs, providing a preconcentration effect nearby the boundary of the zones. However, neutral analytes cannot be concentrated at the early stage of developing the stacking techniques, since they cannot move on the basis of electrophoresis. For the preconcentration of neutral analytes, Liu and coworkers developed a stacking technique containing sodium dodecyl sulfate (SDS) micelle in CE. ${ }^{27}$ In the reported micelle-assisted stacking, a 75-fold enhancement based on the peak area could be achieved. This idea was advanced by Quirino et al:: normal stacking mode $(\mathrm{NSM}),{ }^{28}$ reversed electrode polarity stacking mode (REPSM), ${ }^{29}$ stacking with reverse migrating micelles (SRMM), ${ }^{30}$ stacking using reverse migrating micelles and a water plug (SRW), ${ }^{31}$ and field-enhanced sample injection with reverse migrating micelles $\left(\right.$ FESI-RMM) ${ }^{32}$ were developed for the preconcentration of neutral analytes. In particular, SRW and FESI-RMM could easily attain over 100-fold sensitivity enhancements in CE by injecting a water plug to enhance the electric field. However, the effect of the water plug on the resolution has not been described in detail, since it is difficult to observe the preconcentration and separation processes in the $\mathrm{CE}$ analysis directly. In MCE, a migration process of analytes can be observed ${ }^{20}$ but the introduction of a water plug is very difficult in conventional MCE employing a microchip equipped with a cross-channel injector, since it can inject only one sample solution into the separation channel. Thus, the application of SRW to MCE has not been reported; nevertheless, a microscopic observation in MCE allows researchers to obtain real time monitoring of the process in the MCE analysis.

In the present work, we investigated an effect of injecting a low-conductivity (low-C) zone, which worked as like the 
deionized water plug described in the previous report, into the separation channel by using the formally fabricated microchips with T- and cross-channel configurations (T-cross chip). ${ }^{33}$ The migration behavior of the analytes in the low-C zone in microchip micellar electrokinetic chromatography (MCMEKC) was observed at various detection points by laser-induced fluorescence (LIF) to reveal FASS-MCMEKC with the low-C zone. The effects of the low-C zone on the preconcentration and resolution were also evaluated by investigating the migrating velocity of the model analytes with various values of the retention factor.

\section{Experimental}

\section{Chemicals}

Sulforhodamine 101 (SR101) was purchased from MP Biomedicals (Irvine, CA), sulforhodamine B (SRB) and rhodamine $\mathrm{B}(\mathrm{RB})$ from Tokyo Chemical Industry (Tokyo, Japan), methanol and sodium dodecyl sulfate (SDS) from Nacalai Tesque (Kyoto, Japan). All reagents were of analytical or HPLC grade. Background solutions (BGSs) were prepared by the dilution of a stock SDS solution $(0.5 \mathrm{M})$ with an appropriate phosphate buffer. Methanol was added into the prepared BGS so as to reduce the excessively strong interaction between the micelle and the test analytes. The BGSs were prepared every day to keep the reproducibility as reported previously. ${ }^{30-32,34}$ Sample solution (S) was diluted by deionized water purified by using a Direct-Q System (Nihon Millipore, Japan), and filtered through a $0.45-\mu \mathrm{m}$ pore membrane filter prior to use.

\section{Instrumentation}

T-cross chips made of quartz were fabricated by the conventional photolithographic techniques, as reported previously. ${ }^{33}$ The MCE experiment was performed with a five channel-type high-voltage power supply (Shimadzu, Kyoto, Japan). The detection of analytes in MCE was conducted with a conventional LIF scheme (excitation, $532 \mathrm{~nm}$; emission, $600 \mathrm{~nm}$ ). ${ }^{26,33} \mathrm{CE}$ experiments were performed on a commercial CE instrument (Agilent Technologies, Palo Alto, CA) for determining the fundamental parameter of the analytes.

\section{Procedure}

The T-cross chip was employed to introduce the low-C and S zones into the separation channel by applying programed voltages, as shown in Fig. 1. Although deionized water was filled in the water reservoir (W), "deionized water" could not be introduced into a separation channel under the experimental condition, because the slow cathodic EOF was generated in the channel. During the loading step, as shown in Fig. 1a, however, the low-C zone was generated between the $\mathrm{W}$ and waste reservoirs due to both no supply of the ionic species from the $\mathrm{W}$ reservoir and electrophoretic migration of the anionic species in the channels. At the same time, the offset channel was filled with the S. In Fig. 1b, the low-C zone was also generated in the separation channel by switching the voltages. When the separation voltages were applied to the microchannels, the analytes in the $\mathrm{S}$ solution located in the offset channel was incorporated by the micelle from the BGS reservoir, and then moved into the separation channel (Fig. 1c). As a result, on-line sample preconcentration by FASS-MCMEKC with the low-C zone was expected to be carried out in the separation channel. Finally, the concentrated $\mathrm{S}$ was separated due to the principle of MEKC, and was detected by LIF. In conventional MCMEKC, (a) loading

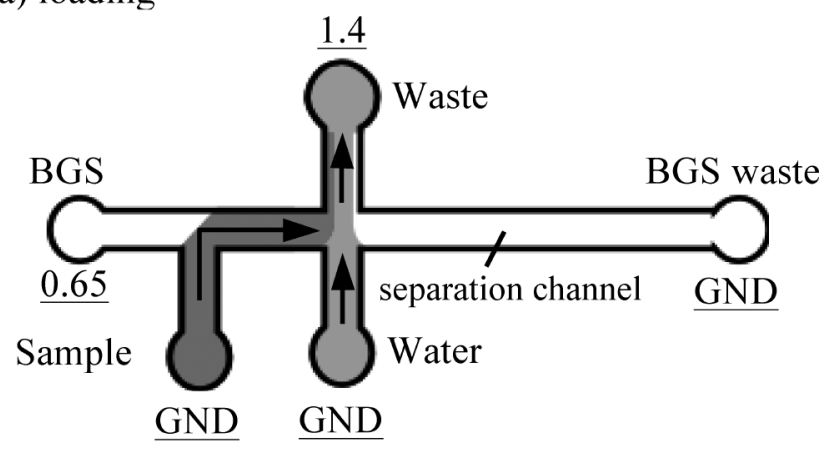

(b) low-C plug injection

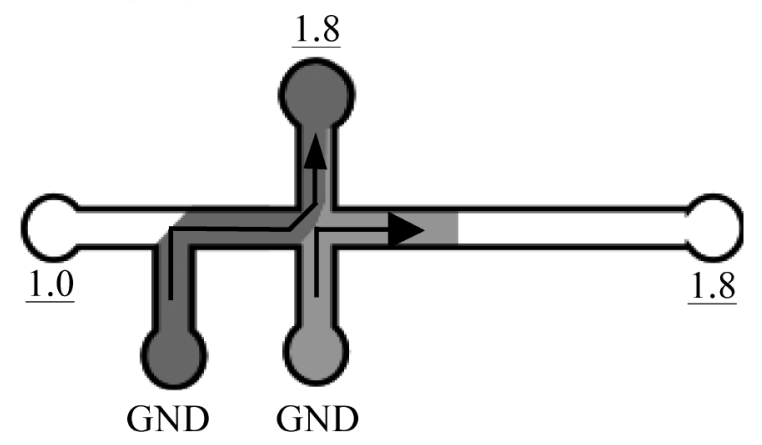

(c) sample injection and separation

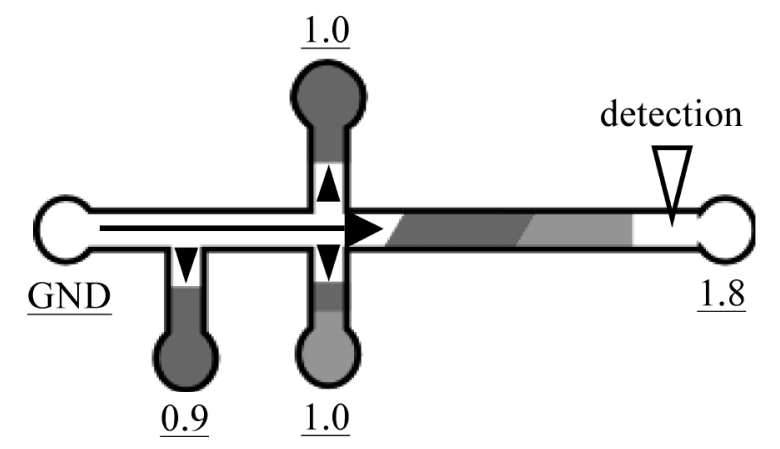

Fig. 1 Injection processes on the T-cross chip. The underlined values are the applied voltage $(\mathrm{kV})$; GND means ground $(0 \mathrm{kV})$.

pinched injection (PI) of sample solution was carried out, as previously reported. ${ }^{33}$ Electropherograms were obtained by measuring the fluorescence intensity at the various detection points.

\section{Concentration process of FASS with the low-C zone}

Schematics of the FASS with a low-C zone under an acidic condition are briefly illustrated in Fig. 2. In FASS-MCMEKC under the acidic condition, a long $\mathrm{S}$ plug is introduced into the separation channel after injection of the low-C zone. Since the local electric field strength in the low-C zone is enhanced by the lower conductivity of the zone than that in the high-C BGS zone, as shown in Fig. 2a, a local velocity of hydrophobic analytes incorporated into the anionic micelle in the low-C zone $\left(v_{\mathrm{S}, \text { low-C}}\right)$ becomes faster than that in the $\mathrm{S}$ zone $\left(v_{\mathrm{S}, \mathrm{S}}\right)$ (Fig. 2b). When the micelle and incorporated $\mathrm{S}$ reaches the boundary between the low-C and BGS zones, the migrating velocity of the micelle is decelerated by the decrease in the electric field, due to the higher conductivity of the BGS. Hence, the decrease in the 

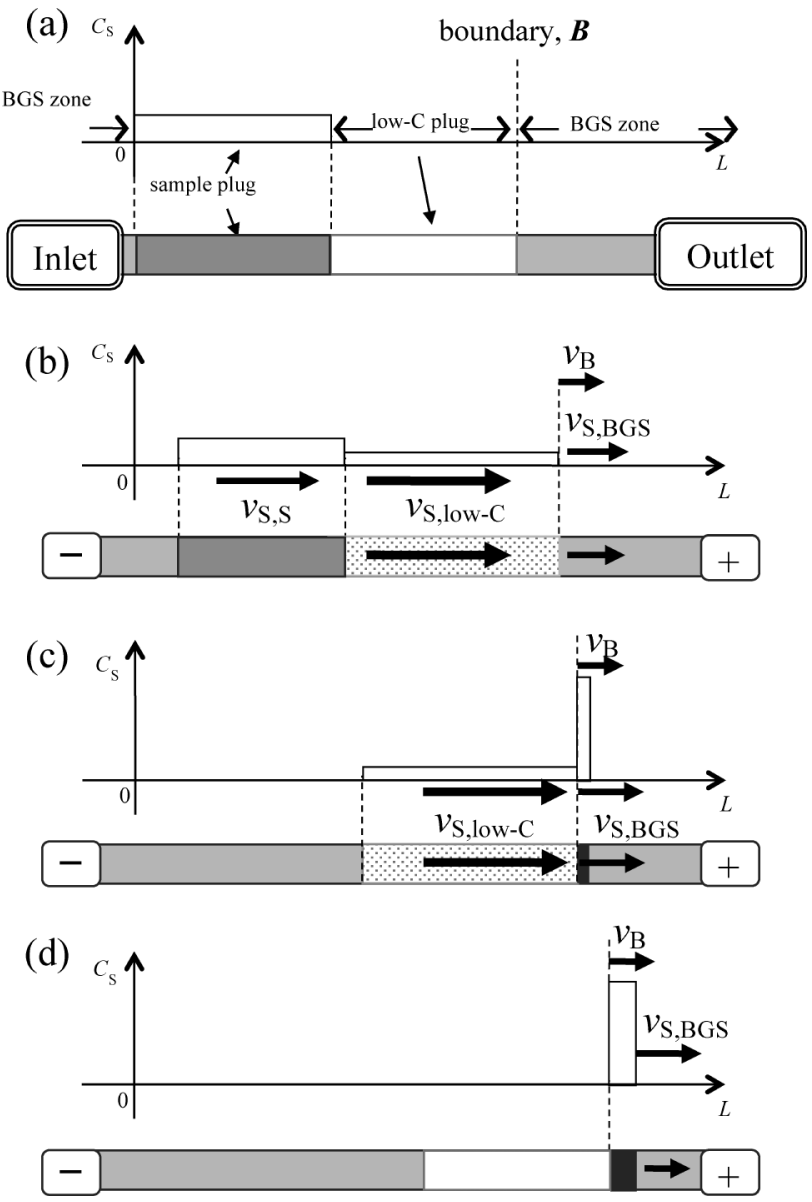

Fig. 2 Schematics of the stacking process with a low-C zone. (a) Initial condition, (b) start of the preconcentration, (c) during stacking, (d) finish of stacking. $v_{\mathrm{B}}$, moving velocity of the boundary; $v_{\mathrm{S}, \mathrm{S}}, v_{\mathrm{S}, \text { low-C }}$, and $v_{\mathrm{S}, \mathrm{BGS}}$, apparent velocities of the analytes in the $\mathrm{S}$, low-C, and BGS zones, respectively.

velocity of analytes in the BGS ( $\left.v_{\mathrm{S}, \mathrm{BGS}}\right)$, and the $\mathrm{S}$ incorporated into the SDS micelle is then concentrated nearby the boundary on the basis of FASS (Figs. 2c and 2d). Finally, the concentrated analytes are separated by MEKC in the BGS.

\section{Results and Discussion}

Confirmation of injection of the low-conductivity zone

To apply an on-line sample preconcentration using a low-C zone to MCMEKC, a zone of which the conductivity was lower than those of the sample and the background solutions must be introduced in front of the $\mathrm{S}$ zone under the reverse migration mode with a suppressed EOF, as shown in Fig. 2. To confirm the injection of the low-C zone, the long $\mathrm{S}$ zone was introduced into the separation channel after applying the voltages shown in Fig. $1 \mathrm{~b}$ for $3 \mathrm{~s}$. Figure 3 shows the typical electropherograms at the different detection points when a $\mathrm{W}$ port was filled with a BGS or deionized water. An anionic rhodamine dye, SRB, was used as the test analyte, since the hydrophilic interaction between the micelle and SRB is so strong that the electrophoretic migration of SRB has a slight influence on the stacking effect in MCMEKC. When the $\mathrm{W}$ port was filled with the BGS (Fig. 3a), SRB started to be stacked immediately after injection of the $\mathrm{S}$ into the separation channel filled with the BGS on the basis of
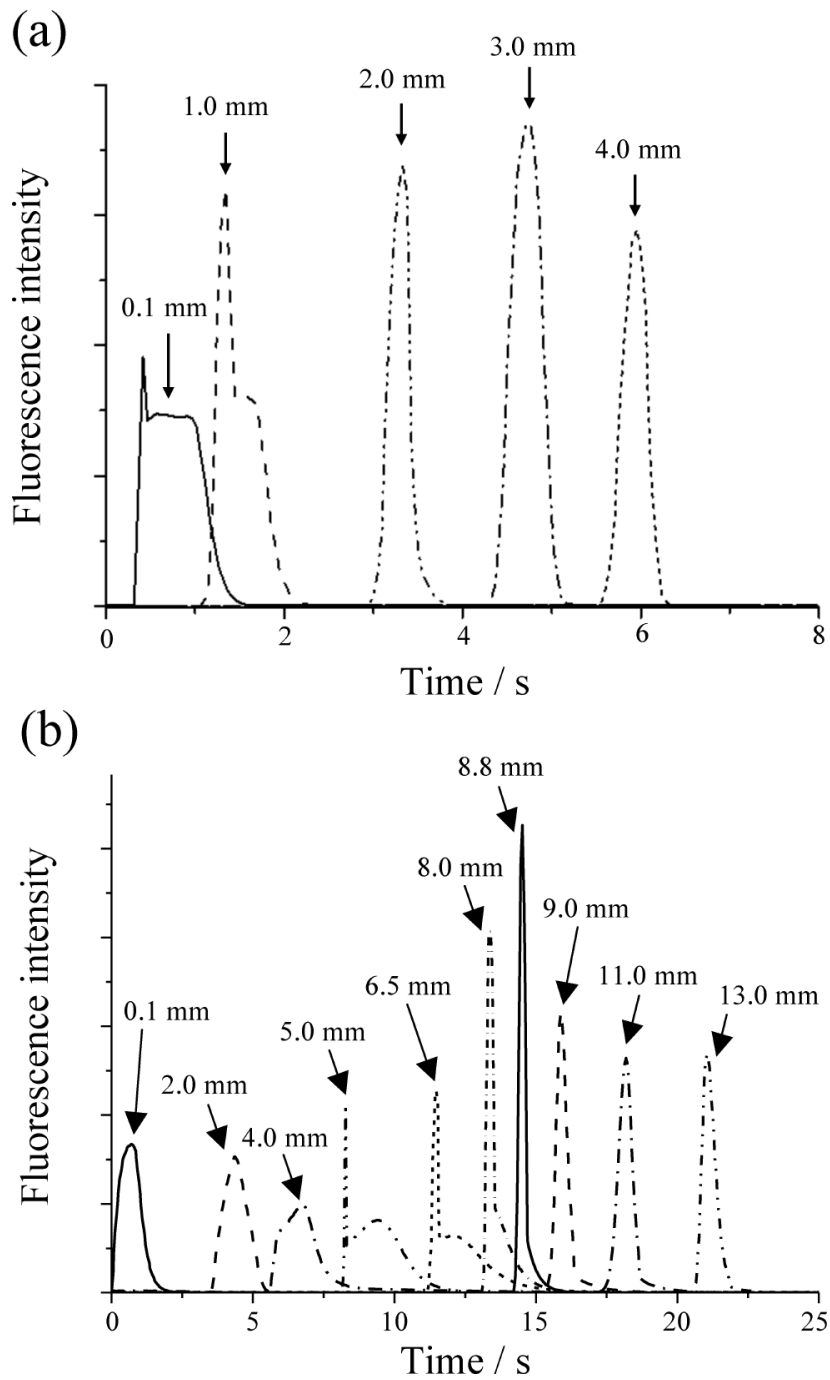

Fig. 3 Observation of the stacking processes in FASS-MCMEKC without/with partial injection of the low-C zone. BGS, $50 \mathrm{mM}$ SDS in $50 \mathrm{mM}$ phosphate buffer ( $\mathrm{pH}$ 2.2). W: (a) BGS, (b) deionized water; S. $10 \mu \mathrm{M}$ SRB prepared in the 100 -fold diluted BGS by the deionized water.

FASS, due to the difference in the conductivities between the $S$ and BGS zones. After stacking, the concentrated sample band was broadened by molecular diffusions of the micelle and the sample molecules. The focusing and broadening of the sample zone indicates that the preconcentration of the samples based on FASS was successfully conducted in MCE. On the other hand, a different behavior of stacking was observed, as shown in Fig. 3b, when deionized water was poured into the $\mathrm{W}$ port. At the detection points of $0.1-4.0 \mathrm{~mm}$, the shapes of the peaks were distorted and broadened without focusing. At the detection point of $5.0 \mathrm{~mm}$, a complicated peak with both sharp and broadened zones was observed, indicating that the analytes started to be stacked nearby there. The peak height of the concentrated zone became higher from 5.0 to $8.8 \mathrm{~mm}$ by the progress of stacking, the almost all of analytes were then focused at a detection point of $8.8 \mathrm{~mm}$. After finishing the preconcentration, the concentrated sample band was broadened by molecular diffusion at detection points of $8.8-13.0 \mathrm{~mm}$. It was also observed that the beginning point of stacking was delayed from 5.0 to $20.0 \mathrm{~mm}$ upon increasing the injection time of the low-C zone $\left(t_{\text {inj,low-C }}\right)$ from 1.0 to $5.0 \mathrm{~s}$. This lag of the 
(a) PI-MCMEKC

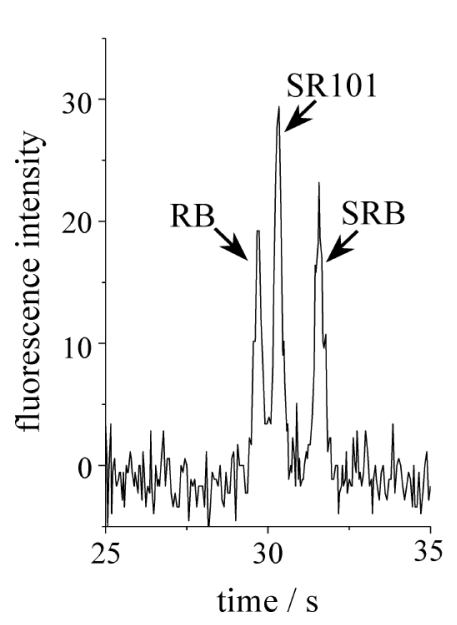

(b) stacking without low-C

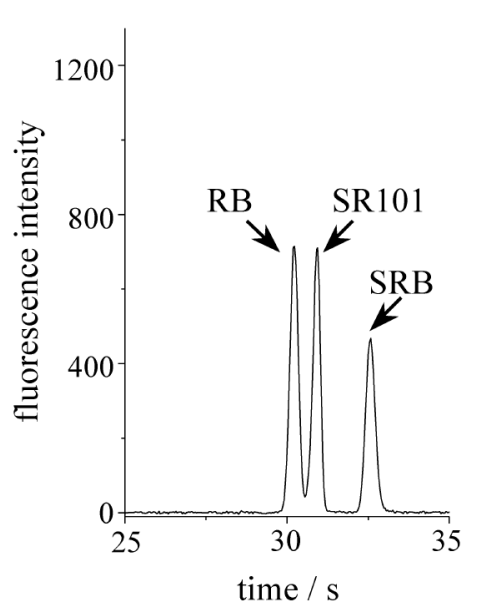

(c) stacking with low-C

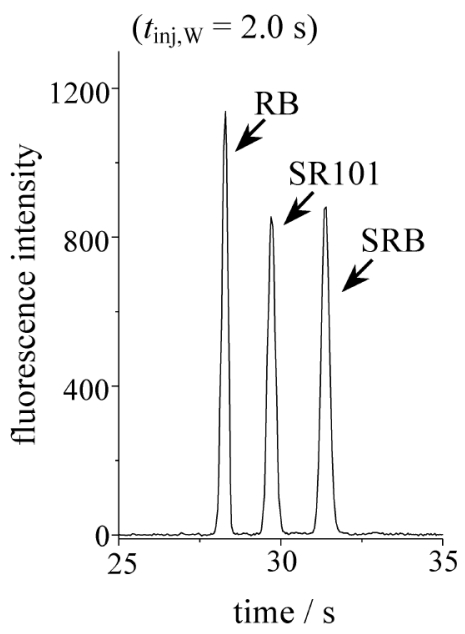

Fig. 4 Effect of the low-C zone on the separation and preconcentration efficiencies. (a) Conventional PI-MCMEKC. Injection time of the low-C zone: (b) 0, (c) $2.0 \mathrm{~s}$. BGS, $20 \mathrm{mM}$ SDS in $50 \mathrm{mM}$ phosphate buffer (pH 2.4)/10\% (v/v) methanol; sample, $1 \mu \mathrm{M}$ RB, SR101 and SRB prepared in the 40fold diluted BGS.

beginning point of stacking suggests generation of the low-C zone, where the conductivity was lower than those of the $\mathrm{S}$ and BGS zones. In addition, the dependence of the lag of stacking on $t_{\text {inj,low-C }}$ indicates that the length of the low-C zone is controllable. Hence, it was confirmed that the introduction of the low-C zone was conducted under the experimental condition.

To investigate these phenomena, the values of the electrophoretic mobility of the anionic species were evaluated by the conventional CE analysis. As a result, those of the SDS micelle, SRB and phosphate ions were calculated to be $c a$. -3.6 , -2.2 , and $-1.7--2.9 \times 10^{-4} \mathrm{~cm}^{2} \mathrm{~V}^{-1} \mathrm{~s}^{-1}$ at $\mathrm{pH} 2.1-2.9$, respectively. Since these values were higher than the electroosmotic mobility of $5.8-7.8 \times 10^{-5} \mathrm{~cm}^{2} \mathrm{~V}^{-1} \mathrm{~s}^{-1}$ under the experimental condition, it was confirmed that these anions could also migrate toward the anode against the suppressed EOF under the acidic condition. In the loading and low-C plug injection steps, therefore, anionic species are not supplied from the $\mathrm{W}$ reservoir filled with deionized water, whereas cationic species migrate toward the $\mathrm{W}$ port. Furthermore, the increasing intensity of the peak upon increasing the effective length means that the stacking boundary also moves toward the anode against the cathodic EOF. Compared to the conventional SRMM and SRW, on the other hand, the reported reversal of the migrating direction of the stacking boundary was not observed under this experimental condition. The detailed mechanism of the reversal was not reported in previous papers, ${ }^{30,31}$ but it is supposed that the extremely low ionic strength of the deionized water and the sample zones generate a temporary enhanced cathodic EOF. ${ }^{35}$ Hence, it was suggested that the ionic strength of the low-C zone is not small enough to generate the temporary enhanced EOF, of which the velocity was faster than electrophoretic migration of the anionic micelle. Consequently, it is clarified that the low-C zone can be formed in the separation channels by migration of the anionic species in order to keep electroneutrality principle.

\section{Effect of the low-C zone on preconcentration and separation}

To evaluate the effect of the low-C zone on preconcentration and separation, FASS-MCMEKC analyses of rhodamine dyes were conducted with partial injection of the low-C zone. In
Table 1 Effect of the injection time of the low-C zone on $\mathrm{SEF}_{\text {height }}$ and $R_{\mathrm{S}}{ }^{\mathrm{a}}$

\begin{tabular}{ccccccc}
\hline & \multicolumn{3}{c}{$\mathrm{SEF}_{\text {height }}$} & & \multicolumn{3}{c}{$R_{\mathrm{S}}$} \\
\cline { 2 - 4 } \cline { 6 - 7 } & RB & SR10w-C1 & SRB & & RB/SR101 & SR101/SRB \\
\hline 0.0 & 26 & 24 & 17 & & 1.4 & 3.2 \\
1.0 & 38 & 29 & 31 & & 2.3 & 3.2 \\
2.0 & 41 & 26 & 31 & & 3.3 & 3.4 \\
3.0 & 41 & 28 & 33 & & 3.4 & 2.7 \\
\hline
\end{tabular}

a. BGS, $20 \mathrm{mM}$ SDS in $50 \mathrm{mM}$ phosphate buffer (pH 2.4)/10\% (v/v) methanol; sample, $1 \mu \mathrm{M} \mathrm{RB}$, SR101 and SRB dissolved in 40-fold diluted BGS.

addition, conventional PI- and FASS-MCMEKC analyses were also carried out. Typical electropherograms were obtained, as shown in Fig. 4. In conventional PI-MCMEKC, RB and SR101 could not be completely separated due to their strong interactions with the SDS micelle. The obtained peak heights of the rhodamine dyes were 20 - 30, as shown in Fig. 4a. Under the stacking conditions with and without injecting low-C zone (Figs. $4 \mathrm{~b}$ and $4 \mathrm{c}$ ), the peak heights were increased as compared to the conventional PI-MCMEKC. To estimate the effect of preconcentration, the sensitivity enhancement factor $\left(\mathrm{SEF}_{\text {height }}\right)$ was calculated from the following equation:

$$
\mathrm{SEF}_{\text {height }}=\frac{C_{\mathrm{PI}}}{C_{\text {stack }}} \times \frac{h_{\text {stack }}}{h_{\mathrm{PI}}},
$$

where $C$ and $h$ are the sample concentration and the peak height, respectively. The subscripts PI and stack mean that these values are obtained in conventional PI-MCMEKC and FASS-MCMEKC, respectively. The obtained values of $\mathrm{SEF}_{\text {height }}$ and resolution $\left(R_{\mathrm{S}}\right)$ with varying $t_{\text {inj,low-C }}$ are summarized in Table 1 . Comparing $\mathrm{SEF}_{\text {height, }}$ it was suggested that the preconcentration efficiency with the low-C zone was slightly higher than that without one. Thus, it was indicated that the introduction of the low-C zone proved to be advantageous to the preconcentration of these analytes. It was also found that the increase in $t_{\text {inj,low-C }}$ did not affect the obtained $\mathrm{SEF}_{\text {height }}$ very much under the experimental 

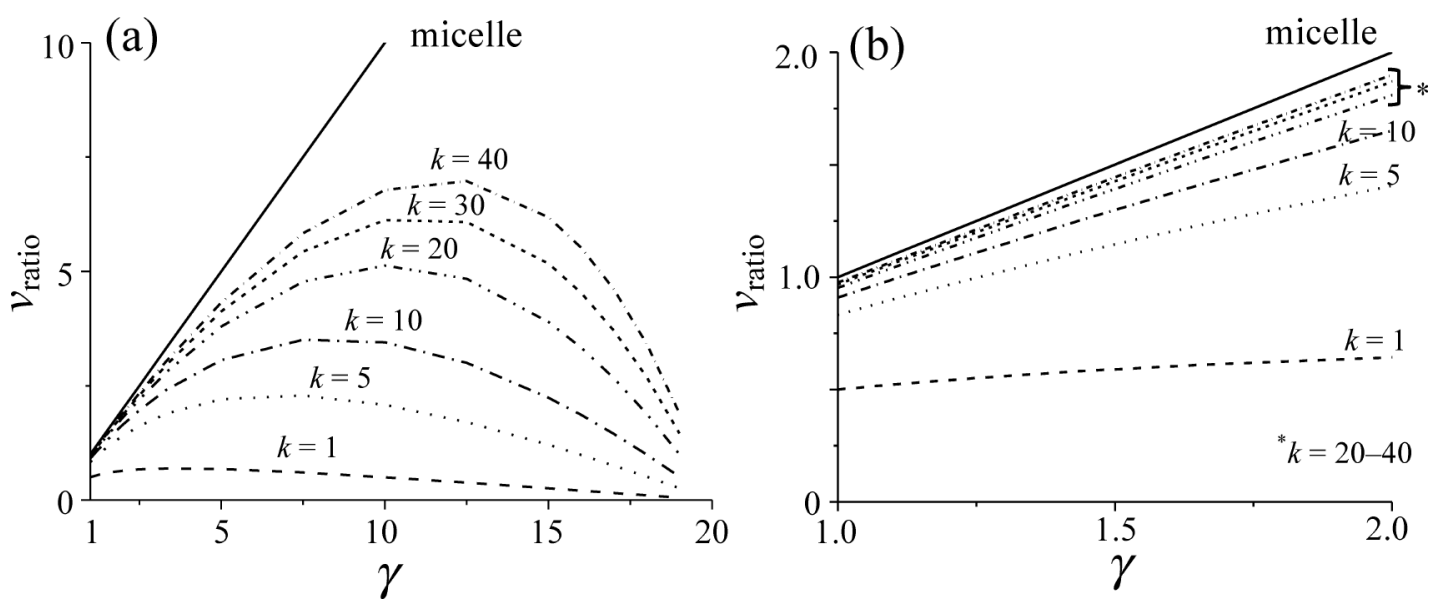

Fig. 5 Plot of $\gamma$ versus $v_{\text {ratio }}$ for the SDS micelle and model analytes with various $k$. (a) BGS, $50 \mathrm{mM}$ SDS in a conventional buffer; low-C solution, $\gamma$ times diluted BGS with deionized water. Solid line, $v_{\text {ratio }}$ of ionic micelle; broken lines, apparent $v_{\text {ratio }}$ of neutral analytes with $k$ of $1-40$. (b) Enlarged drawing of Fig. 5 a.

condition.

Regarding the separation, the $R_{\mathrm{S}}$ for $\mathrm{RB}$ and SR101 was considerably increased from 1.4 to 3.3 upon increasing $t_{\text {inj,low-C }}$ from 1.0 to $2.0 \mathrm{~s}$. With injecting the low-C zone for $3.0 \mathrm{~s}, R_{\mathrm{S}}$ for the RB/SR101 separation was increased from 2.3 to 3.4 , whereas that for SR101/SRB was decreased from 3.2 to 2.7 . Broad peaks were observed due to insufficient separation with injecting the low-C zone over $4.0 \mathrm{~s}$, suggesting unfinished stacking due to a lag of the start of stacking. These results indicated that the low-C zone can affect not only preconcentration based on FASS, but also separation based on MEKC.

Migration behaviors of micelle and samples in the low-C zone

In MEKC, a retention factor $(k)$ is one of the most important parameters that affect the separation of analytes. Under the experimental condition, the concentration of the SDS micelle in the low-C zone should become smaller than that in the $\mathrm{S}$ and BGS zones due to the acceleration of the migration of the anionic micelle according to the enhanced local electric field in the low-C zone. To investigate the effect of the partial introduction of the low-C zone on the MCMEKC separation and FASS preconcentration, the variation of the apparent velocities of the analytes should be discussed. The apparent velocity of the neutral compounds incorporated into an ionic micelle $\left(v_{\mathrm{S}, \mathrm{mc}, \mathrm{app}}\right)$ is defined as follows:

$$
\begin{aligned}
& v_{\mathrm{S}, \mathrm{mc}, \mathrm{app}}=\frac{k}{k+1} v_{\mathrm{mc}}+\frac{1}{k+1} v_{\mathrm{EOF}, \mathrm{ave}} \\
& =\frac{k}{k+1}\left(v_{\mathrm{mc}, \mathrm{ep}}+v_{\mathrm{EOF}, \mathrm{ave}}\right)+\frac{1}{k+1} v_{\mathrm{EOF}, \mathrm{ave}} \\
& =\frac{1}{k+1} \times E \times \mu_{\mathrm{mc}}+v_{\mathrm{EOF}, \mathrm{ave}}, \\
& k=K \bar{v}\left(C_{\text {micelle }}-\mathrm{CNC}\right),
\end{aligned}
$$

where $v^{*}{ }_{\mathrm{mc}}, v_{\mathrm{EOF}, \mathrm{ave}}, E$ and $\mu_{\mathrm{mc}}$ are the effective velocity of the micelle, the averaged velocity of the EOF, the electric field and electrophoretic mobility of the micelle, respectively; $K, \bar{v}$, and $\mathrm{CMC}$ are the distribution coefficient, partial specific volume, and critical micellar concentration, respectively. Equations (2) and (3) clearly tell that the apparent velocity of the analyte is changed by the variation of $C_{\text {micelle }}$. Hence, the decrease in
$C_{\text {micelle }}$ in the low-C zone should reduce the retentions of the analytes.

To evaluate the effect of the variation of $C_{\text {micelle, }}$, the ratios of the conductivity in the BGS and low-C zones $(\gamma)$ and those of the migrating velocities $\left(v_{\text {ratio }}\right)$ of the micelle/analytes were calculated as follows:

$$
\begin{gathered}
\gamma=(\text { conductivity of BGS }) /(\text { conductivity of low-C zone }), \\
v_{\text {ratio }}=\underset{\text { (migrating velocity in low-C zone) } /}{\text { (migrating velocity in BGS) }}
\end{gathered}
$$

It is assumed that the $\mu_{\mathrm{mc}}$ of the micelle is constant in order to simplify the equation, since it has been previously reported that the concentration of the surfactant causes micelles few changes in the mobility. ${ }^{36}$ The difference in the electric field between the BGS and the low-C zones is proportional to $\gamma$, providing a linear increase in $v_{\text {ratio }}$ of the micelle upon increasing $\gamma$ in the assumption (Fig. 5). In the conventional stacking of ionic compounds, since they can also migrate due to their own electrophoretic mobility, a higher $\gamma$ provides a good efficiency of the preconcentration. In MCMEKC of neutral compounds, on the other hand, the decrease in $C_{\text {micelle }}$ by acceleration in the low-C zone leads to decreases in the $k$ values of the analytes. When the initial $C_{\text {micelle }}$ and CMC are 50 and $2.5 \mathrm{mM}, v_{\text {ratio }}$ of the analytes with $k$ of 40 is calculated to be 1.9 at a $\gamma$ of 19 , whereas that at a $\gamma$ of 10 is approximately 6.8. Concerning the less hydrophobic analytes with small $k$ below 1.0, Fig. 5a indicates that the stacking effect was not obtained so much. When $C_{\text {SDS }}$ is lower than CMC, neutral analytes cannot move in the zone, leading to the no stacking effect. These calculations clearly reveal two characteristics concerning the preconcentration of the analytes with the low-C zone: The concentration of SDS is decreased by acceleration due to the stacking effect in a low-C zone, providing a positive correlation between the stacking efficiency and $\gamma$ at the low $\gamma$ condition, and a negative correlation at the high $\gamma$ condition, as shown in Fig. 5a. Hence, the highest stacking efficiency is obtained by injecting the low-C zone with a moderate $\gamma$ in the low-C zone. The other is that the analytes with a higher $k$ are more concentrated as compared to that with a smaller $k$ according to the larger $v_{\text {ratio }}$.

Regarding the effect of the low-C zone on MEKC separation, 
the resolution between RB and SR101 was improved by partial injection of the low-C zone as shown in Fig. 4 and Table 1. It is well-known that the analytes of which the $k$ values are $0.1-10$ are well resolved in MEKC. On the other hand, highly hydrophobic analytes with lager $k$ values than 10 cannot be separated well, since the strongly retained compounds migrate at almost the same velocity as the micelle, as shown in Fig. 5b. Hence, the highly retained $\mathrm{RB}$ and SR101, of which $k$ values were over 20, were not resolved well under MCMEKC conditions. In MCMEKC with the low-C zone, on the other hand, a dilution of the SDS in the low-C zone weakens the retention of the hydrophobic analytes. As a result, the differences in the apparent velocities of the hydrophobic analytes become large at a $\gamma$ of 10, as compared to those of 1.0 (Fig. 5a). Thus, it was found that the injection of the low-C zone could improve the resolution of highly hydrophobic compounds by reducing their $k$ values. However, the separation of the moderately hydrophobic compounds, such as SRB, might be worse by reducing the retention (Table 1). Consequently, it was newly clarified that the introduction of the low-C zone is advantageous for both the preconcentration and separation of highly hydrophobic compounds.

\section{Conclusions}

In this study, the application of partial injection of the low-C zone to FASS-MCMEKC was demonstrated by employing a fabricated T-cross chip. The process of stacking was directly observed by fluorescence imaging, revealing that the low-C zone could be generated in the separation channel. Evaluating the effect of the partial injection of the low-C zone on the retention of the analytes by the SDS micelle clarified that the injected low-C zone is advantageous for both the preconcentration and separation of highly hydrophobic compounds. Consequently, these results indicated that controlling the injection length and conductivity of the low-C zone provides adjustments to both the sensitivity enhancement and improvement of the resolution, not only in MCE, but also in CE.

\section{Acknowledgements}

The authors thank Dr. Hidenori Nagai, Dr. Shin-ichi Wakida, and Dr. Junji Nishii (National Institute of Advanced Industrial Science and Technology, AIST, Japan) for fabricating the microchips. The authors are grateful to the Grant-in-Aid for Young Scientists (B) (Nos. 22750070 and 24750068) from Japan Society for the Promotion of Science (JSPS). This work has been supported in part by the Grant-in-Aid for Scientific Research (No. 24350039) form JSPS. This development was also supported in part by SENTAN, JST.

\section{References}

1. A. Manz, N. Graber, and H. M. Widmer, Sens. Actuators, B, 1990, $1,244$.

2. J. Khandurina and A. Guttman, J. Chromatogr., A, 2002, 943, 159.

3. T. Vilkner, D. Janasek, and A. Manz, Anal. Chem., 2004, 76,3373 .
4. A. Manz, D. J. Harrison, E. Verpoorte, J. C. Fettinger, A. Paulus, H. Lüdi, and H. M. Widmer, J. Chromatogr., 1992, 593, 253.

5. S. C. Jacobson, R. Hergenröder, L. B. Koutny, R. J. Warmack, and J. M. Ramsey, Anal. Chem., 1994, 66, 1107.

6. M. C. Breadmore, Electrophoresis, 2007, 28, 254.

7. S. L. Simpson Jr., J. P. Quirino, and S. Terabe, J. Chromatogr., A, 2008, 1184, 504.

8. K. Sueyoshi, F. Kitagawa, and K. Otsuka, J. Sep. Sci., 2008, $31,2650$.

9. M. C. Breadmore, J. R. E. Thabano, M. Dawod, A. A. Kazarian, J. P. Quirino, and R. M. Guijt, Electrophoresis, 2009, 30, 230.

10. F. Kitagawa, T. Kawai, K. Sueyoshi, and K. Otsuka, Anal. Sci., 2012, 28, 85.

11. J. D. Ramsey and G. E. Collins, Anal. Chem., 2005, 77, 6664.

12. Y.-C. Wang, A. L. Stevens, and J. Han, Anal. Chem., 2005, 77, 4293

13. S. M. Kim, M. A. Burns, and E. F. Hasselbrink, Anal. Chem., 2006, 78, 4779.

14. S. Song, A. K. Singh, and B. J. Kirby, Anal. Chem., 2004, $76,4589$.

15. R. S. Foote, J. Khandurina, S. C. Jacobson, and J. M. Ramsey, Anal. Chem., 2005, 77, 57.

16. D. S. Burgi and R.-L. Chien, Anal. Chem., 1991, 63, 2042.

17. S. C. Jacobson and J. M. Ramsey, Electrophoresis, 1995, 16,481

18. B. Jung, R. Bharadwaj, and J. G. Santiago, Electrophoresis, 2003, 24, 3476.

19. J. P. Quirino and S. Terabe, Science, 1998, 282, 465.

20. Y. Sera, N. Matsubara, K. Otsuka, and S. Terabe, Electrophoresis, 2001, 22, 3509.

21. Y. Liu, R. S. Foote, S. C. Jacobson, and J. M. Ramsey, Lab Chip, 2005, 5, 457.

22. F. Foret, E. Szoko, and B. L. Karger, J. Chromatogr., 1992, $608,3$.

23. M. R. Mohamadi, N. Kaji, M. Tokeshi, and Y. Baba, Anal. Chem., 2007, 79, 3667.

24. S. Hjertén and M.-D. Zhu, J. Chromatogr., 1985, 346, 265.

25. O. Hofmann, D. Che, K. A. Cruickshank, and U. R. Müller, Anal. Chem., 1999, 71, 678.

26. K. Sueyoshi, F. Kitagawa, and K. Otsuka, Anal. Chem., 2008, 80, 1255.

27. Z. Liu, P. Sam, S. R. Sirimanne, P. C. McClure, J. Grainger, and D. G. Patterson, J. Chromatogr., A, 1994, 673, 125.

28. J. P. Quirino and S. Terabe, J. Chromatogr., A, 1997, 781, 119.

29. J. P. Quirino and S. Terabe, J. Chromatogr., A, 1997, 791, 255.

30. J. P. Quirino and S. Terabe, Anal. Chem., 1998, 70, 149.

31. J. P. Quirino and S. Terabe, J. Chromatogr., B, 1998, 714, 29.

32. J. P. Quirino and S. Terabe, Anal. Chem., 1998, 70, 1893.

33. K. Sueyoshi, H. Nagai, S. Wakida, J. Nishii, F. Kitagawa, and K. Otsuka, Meas. Sci. Technol., 2006, 17, 3154.

34. J. L. Kurz, J. Phys. Chem., 1962, 66, 2239.

35. T. Kawai, K. Sueyoshi, F. Kitagawa, and K. Otsuka, Anal. Chem., 2010, 82, 995.

36. S. Terabe, K. Otsuka, and T, Ando, Anal. Chem., 1985, 57, 834. 